\title{
Open Problems in Symmetry Analysis
}

\author{
Peter A Clarkson and Elizabeth L Mansfield
}

\begin{abstract}
In this paper we discuss open problems associated with the study of symmetry reductions and exact solutions of differential equations.
\end{abstract}

\section{Introduction}

Nonlinear phenomena have many important applications in the natural and applied sciences. Essentially all the fundamental equations of physics are nonlinear and, in general, are often difficult to solve explicitly. Consequently perturbation, asymptotic and numerical methods are often used, with much success, to obtain approximate solutions; however, there is also much current interest in obtaining exact analytical solutions. These have many mathematical and physical applications, and are usually obtained either by seeking a solution in a special form or, more generally, by exploiting symmetries of the equation. This last provides a method for obtaining exact solutions of a given equation in terms of solutions of lower dimensional equations, in particular, ordinary differential equations.

The classical method for finding symmetries and reductions of partial differential equations is due to Lie (cf. $[\mathbf{5}, \mathbf{6 9}]$ ). Suppose $x, t$ are the independent variables, $u$ the dependent variable, and $\boldsymbol{u}^{(\ell)}$ denotes the set of all the partial derivatives of order $\ell$ of $u$. To apply the classical method to the general $N$ th order partial differential equation

$$
\Delta=\Delta\left(x, t, u, \boldsymbol{u}^{(1)}, \ldots, \boldsymbol{u}^{(N)}\right)=0
$$

we consider the one-parameter Lie group of infinitesimal transformations in $(x, t, u)$ given by

$$
\begin{aligned}
\widetilde{x} & =x+\varepsilon \xi(x, t, u)+\mathcal{O}\left(\varepsilon^{2}\right), \\
\widetilde{t} & =t+\varepsilon \tau(x, t, u)+\mathcal{O}\left(\varepsilon^{2}\right), \\
\widetilde{u} & =u+\varepsilon \phi(x, t, u)+\mathcal{O}\left(\varepsilon^{2}\right),
\end{aligned}
$$

where $\varepsilon$ is the group parameter. Requiring that (1.1) is invariant under this transformation yields an overdetermined, linear system of equations for the infinitesimals $\xi(x, t, u), \tau(x, t, u)$ and $\phi(x, t, u)$. The associated Lie algebra of infinitesimal symmetries is the set of vector fields of the form

$$
\mathbf{v}=\xi(x, t, u) \partial_{x}+\tau(x, t, u) \partial_{t}+\phi(x, t, u) \partial_{u}
$$

where $\partial_{x} \equiv \partial / \partial x$ etc. Though this method is largely algorithmic, it often involves a great deal of tedious algebra and auxiliary calculations which can become virtually unmanageable if attempted manually, and so symbolic manipulation programs have been developed to facilitate the calculations; an excellent survey of the different packages presently available and a discussion of their strengths and applications is given by Hereman [38].

Once a symmetry group is known, the reduction of the equation is obtained by first solving the invariant surface condition

$$
\psi \equiv \xi(x, t, u) u_{x}+\tau(x, t, u) u_{t}-\phi(x, t, u)=0,
$$

using the method of characteristics. This typically yields $u(x, t)=U(x, t, w(z(x, t)))$ where $U$ and $z$ are known functions. Substituting this into (1.1) will then yield an ordinary differential equation for $w$ as a function of $z$, and this is the reduction of (1.1).

in "Geometrical Study of Differential Equations" [Editors J A Leslie and T Robart], Contemporary Mathematics Series, vol. 285, American Mathematical Society, Providence, RI (2001) pp 195-205. 
There have been several generalizations of the classical Lie group method for symmetry reductions. Bluman and Cole [4] proposed the so-called nonclassical method of group-invariant solutions. In this method, the original partial differential equation (1.1) is augmented with the invariant surface condition (1.4). By requiring that the set of simultaneous solutions of (1.1) and (1.4) are invariant under the transformation (1.2), one obtains an overdetermined, nonlinear system of equations, as opposed to a linear system in the classical case, for the infinitesimals $\xi, \tau$ and $\phi$; note these appear in both the transformations (1.2) and the supplementary condition (1.4). The number of determining equations arising in the nonclassical method is smaller than for the classical method, since there are fewer linearly independent expressions in the derivatives of $u$. Since all solutions of the classical determining equations necessarily satisfy the nonclassical determining equations, the solution set may be larger in the nonclassical case. It should be emphasized that the vector fields associated with the nonclassical method do not form a vector space, still less a Lie algebra, since the invariant surface condition (1.4) depends upon the particular reduction. For example, the sum of two nonclassical symmetry operators is not, in general, a symmetry operator at all; similarly the commutator of two nonclassical symmetry operators, or the sum of a classical symmetry operator and a nonclassical symmetry operator are not, in general, symmetry operators.

Subsequently, the nonclassical method was further generalized by Olver and Rosenau [71, 72] to include "weak symmetries" and, even more generally, "side conditions" or "differential constraints". However their framework appears to be too general to be practical and they concluded that: "the unifying theme behind finding special solutions of partial differential equations is not, as is commonly supposed, group theory, but rather the more analytic subject of overdetermined systems of partial differential equations".

Motivated by the fact that symmetry reductions of the Boussinesq equation

$$
u_{t t}+u u_{x x}+u_{x}^{2}+u_{x x x x}=0
$$

were known that are not obtainable using the classical Lie group method, Clarkson and Kruskal [21] developed a direct, algorithmic method for finding symmetry reductions (in the sequel referred to as the direct method), which they used to obtain previously unknown reductions of the Boussinesq equation. The basic idea of the direct method is to seek a solution of a partial differential equation such as (1.1) in the form

$$
u(x, t)=F(x, t, w(z)), \quad z \equiv z(x, t),
$$

and require that $w(z)$ satisfy an ordinary differential equation. This imposes conditions upon $F(x, t, w)$, $z(x, t)$ and their derivatives in the form of an overdetermined system of equations, whose solution yields the desired reductions. Levi and Winternitz [51] subsequently gave a group theoretical explanation of these results by showing that all the new reductions of the Boussinesq equation could be obtained using the nonclassical method of Bluman and Cole [4]. The novel characteristic about the direct method, in comparison to the others mentioned above, is that it involves no use of group theory. We remark that the direct method has certain resemblances to the so-called "method of free parameter analysis" (cf. [37]); though in this latter method, the boundary conditions are crucially used in the determination of the reduction whereas they are not used in the direct method. Additionally ansatz based methods for determining reductions and exact solutions of partial differential equations have been used by Fushchych and co-workers (cf. [35] and the references therein).

The nonclassical method lay dormant for several years, essentially until the papers by Olver and Rosenau $[\mathbf{7 1}, \mathbf{7 2}]$. However, following the development of the direct method there has been renewed interest in the nonclassical method and recently both these methods have been used to generate many new symmetry reductions and exact solutions for several physically significant partial differential equations which represents significant and important progress (cf. $[\mathbf{1 9}, \mathbf{2 2}, \mathbf{2 9}, \mathbf{3 5}]$ and the references therein).

\section{Open Problems}

2.1. When does the classical Lie group method need to be generalized? For some equations, such as the Korteweg-de Vries (KdV) equation

$$
u_{t}+6 u u_{x}+u_{x x x}=0
$$

the symmetry reductions arising from the classical, direct and nonclassical methods coincide [21]. The classical method is usually the simplest to calculate, since all the equations to be solved are linear and a variety of symbolic manipulation programs have been developed that implement the method.

Open Question 2.1. How does one determine a priori which partial differential equations possess symmetry reductions that are not obtainable using the classical Lie group approach? 
2.2. Algorithms and symbolic manipulation programs. A major difficulty in the determination of symmetry reductions by any method is the large quantity of routine calculations that are usually involved, for which symbolic manipulation programs are ideally suited. In order to solve overdetermined systems of partial differential equations (and in particular nonlinear systems) the development of efficient algorithms is required. At present all the symbolic manipulation programs for the direct and nonclassical methods are interactive. For example, the MACSYMA package symmgrp.max $[\mathbf{2 0}]$ can be adapted to generate the determining equations for the nonclassical method [24], though one has to solve the resulting nonlinear system of equations. The use of differential Gröbner bases (cf. [61]) or characteristic sets $[\mathbf{8}, \mathbf{4 2}]$ has made the analysis of overdetermined systems of partial differential equations, such as those arising as the determining equations for classical and nonclassical symmetries, more tractable. Whilst the diffgrob2 [55] package is best used interactively on large or complex systems, nevertheless it has proved effective in solving such overdetermined systems (cf. $[\mathbf{2 3}, \mathbf{2 4}, \mathbf{2 5}, \mathbf{2 6}, \mathbf{2 7}, \mathbf{5 5}, \mathbf{5 6}, \mathbf{5 9}, \mathbf{6 0}, \mathbf{6 2}]$ ). Other symbolic manipulation packages which can handle nonlinear overdetermined systems of partial differential equations are diffalg, distributed as part of the Maple Library, and the Maple package rif, [76], available from http://www.cecm.sfu.ca/ wittkopf/.

For example, Clarkson and Mansfield [23] applied the nonclassical method in conjunction with the method of differential Gröbner bases to the nonlinear heat equation

$$
u_{t}=u_{x x}+f(u)
$$

where $f(u)$ is an arbitrary sufficiently differentiable function. Clarkson and Mansfield determined conditions on $f(u)$ under which symmetries other than the trivial spatial and temporal translational symmetries exist, solved the determining equations for the infinitesimals and thus obtained a complete catalogue of symmetry reductions for the nonlinear heat equation (2.2). In particular a classification of exact solutions of (2.2) for $f(u)=(u-a)(u-b)(u-c)$, with $a, b$ and $c$ constants, appears in [23].

The four nonclassical determining equations for the nonlinear heat equation (2.2) are

$$
\begin{aligned}
& \xi_{u u}=0, \\
& \phi_{u u}-2 \xi_{x u}+2 \xi \xi_{u}=0, \\
& 2 \phi_{x u}-2 \phi \xi_{u}+3 \xi_{u} f+\xi_{t}-\xi_{x x}+2 \xi \xi_{x}=0, \\
& \phi_{t}-\phi_{x x}+\phi_{u} f-f_{u} \phi+2 \phi \xi_{x}-2 \xi_{x} f=0 .
\end{aligned}
$$

In contrast, the four nonclassical determining equations for the nonlinear wave equation

$$
u_{t t}=u_{x x}+f(u)
$$

are

$$
\begin{aligned}
& \left(\xi^{2}-1\right)\left[\left(\xi^{2}-1\right) \xi_{u u}-2 \xi \xi_{u}^{2}\right]=0 \\
& \left(\xi^{2}-1\right)\left[2 \phi \xi \xi_{u u}+2 \xi_{x u}-2 \xi \phi_{u} \xi_{u}+2 \xi \xi_{t u}+\left(\xi^{2}-1\right) \phi_{u u}\right] \\
& \quad-4 \phi \xi^{2} \xi_{u}^{2}-4 \xi^{2} \xi_{t} \xi_{u}-4 \xi \xi_{u} \xi_{x}=0 \\
& 72 \phi_{x} \xi \xi_{x}-2 \phi \phi_{u} \xi_{x}-2 \phi_{t} \xi_{x}+2 f(u) \xi_{x}-2 \phi^{2} \phi_{u} \xi \xi_{u}-2 \phi \phi_{t} \xi \xi_{u}+2 f(u) \phi \xi \xi_{u} \\
& \quad+2 \phi \phi_{x} \xi_{u}-2 \phi \phi_{u} \xi \xi_{t}-2 \phi_{t} \xi \xi_{t}+2 f(u) \xi \xi_{t}+2 \phi_{x} \xi_{t}+2 \phi \phi_{t u} \xi^{2} \\
& \quad+\left(\xi^{2}-1\right)\left[\phi^{2} \phi_{u u}+f(u) \phi_{u}+\phi_{t t}-\phi_{x x}-\phi f^{\prime}(u)\right]=0 \\
& \left(\xi^{2}-1\right)\left[\xi_{x x}-\phi^{2} \xi_{u u}+2 \phi_{t} \xi_{u}-3 f(u) \xi_{u}-\xi_{t t}-2 \phi \xi_{t u}-2 \phi \phi_{u u} \xi-2 \phi_{t u} \xi\right. \\
& \left.\quad-2 \phi_{x u}\right]-2 \xi_{x}^{2}+4 \phi_{u} \xi \xi_{x}+2 \phi^{2} \xi \xi_{u}^{2}+4 \phi \phi_{t} \xi \xi_{u} \\
& \quad+4 \phi \phi_{u} \xi^{2} \xi_{u}+2 \xi \xi_{t}^{2}+2 \phi_{u}\left(\xi^{2}+1\right) \xi_{t}=0 .
\end{aligned}
$$

Whilst the system (2.3-2.6) is relatively straightforward to solve, the system (2.8-2.11) is significantly more difficult, indeed still unsolved. For equations such as the nonlinear wave (2.7) equation and equations involving the Laplace operator, the analysis of the associated overdetermined systems is currently intractable. This is conjectured to be due to symmetries of the systems of determining equations themselves, arising from the fact that the independent variables appear on an equal footing in the original equation. An idea for overcoming this difficulty is to rewrite the overdetermined system in terms of the invariants of the symmetry, effectively dividing the symmetries out before the processes that analyze the system begin.

In particular, one can use the new formulation of the method of moving frames due to Fels and Olver $[33,34]$, with which one can rewrite a system which is invariant under a group action, in terms of the differential invariants. The kinds of calculations involved and the issues that arise are discussed in [58]. 
Open Question 2.2. To develop algorithms to solve overdetermined systems associated with partial differential equations which have a high degree of symmetry.

2.3. Boundary Value Problems. Consider the second-order partial differential equation

$$
\Delta\left(x, t, u, u_{x}, u_{t}, u_{x x}, u_{x t}, u_{t t}\right)=0,
$$

and the boundary conditions

$$
B_{j}\left(x, t, u, u_{x}, u_{t},\right)=0, \quad j=1,2, \ldots, m
$$

on

$$
\Omega_{j}(x, t)=0, \quad j=1,2, \ldots, m .
$$

The standard procedure for applying the classical method (cf. [5]) to the boundary value problem (2.12-2.14) is to require that both equation and boundary conditions are invariant under the one-parameter Lie group of infinitesimal transformations (1.2), i.e. to require that

$$
\begin{aligned}
& \left.\operatorname{pr}^{(2)} \mathbf{v}(\Delta)\right|_{\Delta=0}=0, \\
& \left.\mathbf{v}\left(\Omega_{j}\right)\right|_{\Omega_{j}=0}=0, \quad j=1,2, \ldots, m, \\
& \left.\operatorname{pr}^{(1)} \mathbf{v}\left(B_{j}\right)\right|_{B_{j}=0}=0, \quad \text { on } \quad \Omega_{j}=0, \quad j=1,2, \ldots, m,
\end{aligned}
$$

where $\mathbf{v}$ is given by (1.3). Equation (2.15) is the standard condition for the partial differential equation (2.12) to be invariant under the infinitesimal transformations (1.2), so that (2.16-2.17) impose additional conditions for a symmetry to exist. Thus this procedure necessarily yields a smaller set of symmetries for the boundary value problem $(2.12-2.14)$ than the partial differential equation (2.12). We believe that this is too restrictive and instead one should only require that a subset of the solutions of (2.12), which is determined from the boundary conditions (2.13-2.14), be invariant under the infinitesimal transformations (1.2). As we remarked above, in the "method of free parameter analysis" the boundary conditions are crucially used in the determination of the reduction (cf. [37]).

OPEN QUESTION 2.3. Is there a general procedure, for applying symmetry methods to boundary value problems?

2.4. Symmetry reductions for differential-difference equations. There is much current interest in deriving symmetries of differential-difference equations such as the Toda lattice equation

$$
\frac{\partial^{2} u_{n}}{\partial x \partial t}=\exp \left(u_{n-1}-u_{n}\right)-\exp \left(u_{n}-u_{n+1}\right) \text {. }
$$

There have been two different approaches to the determination of classical Lie symmetry reductions of differential-difference equations, one proposed by Levi and Winternitz $[\mathbf{5 2}, \mathbf{5 3}]$ and another by Quispel, Capel and Sahadevan [75]. Levi and Winternitz [53] have also introduced the concept of conditional symmetries for the Toda lattice equation (2.18).

Essentially, the difference between these two approaches concerns the interpretation of the discrete variable $n$. Levi and Winternitz $[\mathbf{5 2}, \mathbf{5 3}]$ essentially treat $u_{n-1}, u_{n}$ and $u_{n+1}$ as different dependent variables and thus regard the differential-difference equation as a system of partial differential equations for the functions $u_{n}$. On the other hand, Quispel, Capel and Sahadevan [75] treat $n$ as an independent variable (in addition to $x$ and $t$ ) and thus, in effect, treat the differential-difference equation as a differential-delay equation. Both the above methods are computationally complex, and their philosophical foundations open to debate.

A more recent method for obtaining symmetries of difference equations is due to Hydon [43]. Hydon shows that once the symmetries are cast into so-called "characteristic form", there is a very natural translation of all the calculations for symmetries of differential equations, to difference equations. Other recent developments in the study of symmetries of differential-difference equations include $[\mathbf{3 2}, \mathbf{3 9}, \mathbf{4 8}, \mathbf{5 0}]$.

Open Question 2.4. To clarify the relationship of these approaches (if possible) to each other, and to produce a single standard definition of a symmetry of differential-difference (and difference) equations.

OPEn Question 2.5. The study of nonclassical symmetry reductions of differential-difference and difference equations. 
2.5. Potential symmetries. Potential symmetries (sometimes called nonlocal symmetries) are another type of symmetry which have received some attention recently (cf. [3, 5, 47]). The existence of potential symmetries has led to the construction of corresponding invariant solutions as well as to the linearization of nonlinear partial differential equations through non-invertible mappings $[\mathbf{5}, \mathbf{6}]$. In this approach, a partial differential equation is written as a system of partial differential equations.

For example, consider the shallow water wave (SWW) equation

$$
v_{x x t}+\alpha v v_{t}-\beta v_{x} \partial_{x}^{-1} v_{t}-v_{t}-v_{x}=0,
$$

where $\left(\partial_{x}^{-1} f\right)(x)=\int_{x}^{\infty} f(y) \mathrm{d} y$, and $\alpha$ and $\beta$ are arbitrary nonzero constants. Since this equation contains a nonlocal term then in order to undertake symmetry analysis we need to write (2.19) as an analytic equation or system. Five possible ways of doing this are

$$
\begin{aligned}
& u_{x x x t}+\alpha u_{x} u_{x t}+\beta u_{t} u_{x x}-u_{x t}-u_{x x}=0, \\
& \left\{\begin{array}{l}
v_{x x t}+\alpha v v_{t}+\beta w v_{x}-v_{t}-v_{x}=0, \\
w_{x}=v_{t}
\end{array}\right. \\
& \left\{\begin{array}{l}
v_{x x t}+\alpha v v_{t}+\beta w v_{x}-v_{t}-v_{x}=0, \\
v=u_{x} \\
w=u_{t}
\end{array}\right. \\
& \left\{\begin{array}{l}
\psi_{x}=u_{x x x}+\frac{1}{2}(\alpha-\beta) u_{x}^{2}-u_{x}, \\
\psi_{t}=u_{x}-\beta u_{t} u_{x}
\end{array}\right. \\
& \left\{\begin{array}{l}
v_{x x t}+\alpha v v_{t}+\beta u_{t} v_{x}-v_{t}-v_{x}=0, \\
v=u_{x} .
\end{array}\right.
\end{aligned}
$$

Symmetry reductions using the classical and nonclassical methods and exact solutions of these five variants $(2.20-2.24)$ of SWW equation (2.19) are studied in $[\mathbf{2 5}, \mathbf{2 8}]$. Both the classical and nonclassical methods give the same reductions when applied to the system (2.21) as when applied to the scalar counterpart equation (2.20). What is unusual about the calculation for the system (2.21) is the large increase in complexity in moving from a scalar equation to this system. Whilst for both the system and the scalar equation the determining equations for the classical method are of similar complexity (and are all linear) the complexity of the nonclassical method differs wildly. Another difficulty observed was that the reductions obtained using the nonclassical method for the systems (2.21) and (2.22) arise in a very unusual manner, since one has to use a hodograph transformation to solve the equations. Clarkson and Priestley [28] also applied the direct method to the five equations (2.20-2.24) and obtained the same results as using the nonclassical method. The application of the direct method is not entirely straightforward either especially for the systems (2.21-2.24), though it seems to be much easier to implement than the nonclassical method.

Open QUESTION 2.6. How does one determine a priori the most suitable representation, from the point of view of symmetry calculations, of a nonlocal equation such as (2.19)?

2.6. Generalized symmetries. There are a number of exact solutions of partial differential equations that are not obtained using either the direct or nonclassical methods. The two-soliton solutions, and more generally $N$-soliton solutions, of completely integrable equations such as the Boussinesq equation (1.5) are examples. These are standardly obtainable using generalized symmetries (cf. [2]), which are sometimes known incorrectly as Lie-Bäcklund symmetries. Clarkson and Mansfield [24, 25] have shown that some special "two-soliton" solutions arise from nonclassical symmetries.

A genuine nonclassical symmetry (i.e., one that is not purely a classical symmetry) has the property that the associated infinitesimal transformation leaves invariant only a subset of solutions of the original partial differential equation whereas the infinitesimal transformation for a classical symmetry maps the set of all solutions of the partial differential equation into itself. What is the analogous property (if any) for nonclassical generalized symmetries?

Open QUestion 2.7. Do nonclassical, generalized symmetries exist and if so what kind of solutions do they generate?

Since the Boussinesq equation (1.5) possesses such a rich family of nonclassical symmetry reduction solutions, then we believe that this would be a good example to consider.

2.7. Symmetry in Numerical Analysis. Budd and Collins [10] discuss numerical methods which make use of the symmetry in partial differential equations (see also $[\mathbf{1 4}, \mathbf{1 5}, \mathbf{1 6}]$ ). Such numerical methods have the following advantages: 
- If a numerical method reflects the underlying symmetry of the partial differential equation then it will generally admit self-similar solutions as possible solutions. A well-designed method of this type should also inherit many of the stability properties of such solutions. Thus for problems where selfsimilarity reflects the underlying asymptotic behaviour such methods will have good asymptotic behaviour.

- If symmetry governs singularity formation then these methods should perform well.

- Symmetry is associated with conservation laws through Noether's theorem (cf. [69]), in particular if there is an associated Lagrangian. The numerical method may inherit some of the conservation laws.

- Symmetry can play a useful role in the error analysis of numerical methods.

Numerical methods for ordinary differential equations on Lie groups, e.g. those have a time-stepping strategy that ensure that the solution stays on the group, are of much current interest and are classified as so-called geometric integrators (cf. $[\mathbf{1 4}, \mathbf{4 5}, \mathbf{6 3}, \mathbf{6 4}, \mathbf{6 5}]$ ). Recently symmetry has been included in the design of numerical methods for partial differential equations (see, for example, $[\mathbf{9}, \mathbf{1 1}, \mathbf{1 2}, \mathbf{1 3}, \mathbf{3 1}, \mathbf{4 1}]$ ). Methods for including symmetry into numerical integrators for ordinary differential equations are discussed in [45] and include:

- Luck: some Runge Kutta methods preserve the group structure (cf. [77]).

- Iterated commutator methods which are constructed using the commutator of the group (cf. [45, $46,54,78])$.

- Tangent space and related methods which are constructed to stay in the tangent bundle of the Lie group (cf. $[30,67]$ ).

Open Question 2.8. The design and further development of numerical algorithms for ordinary and, in particular, partial differential equations which inherit symmetries of the differential equations including nonclassical symmetries.

2.8. More general methods. As illustrated above, the direct and nonclassical methods have generated many reductions and exact solutions for a number of physically important partial differential equations. What further generalizations are possible? Galaktionov's method of "nonlinear separation" [36] and Olver and Rosenau's method of differential constraints $[\mathbf{7 1}, \mathbf{7 2}]$ are very general methods. In fact, the method of differential constraints can be viewed as the ultimate generalization since it includes (almost) all the known methods for determining special solutions of partial differential equations such as classical group-invariant solutions, nonclassical and weak symmetry reductions, partially invariant solutions, separation of variables solutions etc. They do have a group theoretic interpretation as "generalized weak symmetries" although, as Olver [68] admits, this does not appear to be an overly useful observation. The main difficulty with this approach is that it appears to be too general to practical use.

Open Question 2.9. How does one determine a priori which differential constraints are useful and which ansätze will yield computable solutions?

Generalizations of the direct method include those due to Burdé [17], Galaktionov [36] and Hood [40]. Generalizations of the nonclassical method are discussed by Bluman and Shtelan [7], Burdé [18] and Olver and Vorob'ev [73].

We remark that a generalisation of the classical method of reduction without the transversality requirement is given by Anderson, Fels and Torre [1] (see also article this volume). Finally, the so-called " $\mathcal{C}$ ( symmetries" of M.C. Muriel Patino [74], is in effect a generalised method of reduction for ordinary differential equations. The known examples of reduction not obtainable by the classical method can be obtained this way.

\section{Acknowledgements}

It is a pleasure to thank Mark Ablowitz, George Bluman, Chris Budd, Willy Hereman, Peter Hydon, Arieh Iserles, Martin Kruskal, Clara Nucci, Peter Olver, Greg Reid and Pavel Winternitz for their contributions, helpful comments and illuminating discussions. We thank the referee for helpful comments and pointing out some of the references.

\section{References}

[1] I.M. Anderson, M.E. Fels and C.G. Torre, Commun. Math. Phys., 212 (2000) 653-686.

[2] R.L. Anderson and N.H. Ibragimov, Lie-Bäcklund Transformations in Applications, SIAM, Philadelphia (1979).

[3] G.W. Bluman, Math. Comp. Model., 18 (1993) 1-14. 
[4] G.W. Bluman and J.D. Cole, J. Math. Mech., 18 (1969) 1025-1042.

[5] G.W. Bluman and S. Kumei, Symmetries and Differential Equations, Appl. Math. Sci. vol. 81, Springer-Verlag, Berlin (1989).

[6] G.W. Bluman and S. Kumei, Europ. J. Appl. Math, 1 (1990) 217-223.

[7] G.W. Bluman and V. Shtelen, in Mathematics is for Solving Problems [Eds. L.P. Cook, V. Roytburd and M. Turin], SIAM, Philadelphia, pp. 105-117 (1996).

[8] F. Boulier, D. Lazard, F. Ollivier and M. Petitot, in Proc. ISSAC '95 [Ed. A. Levelt], ACM Press, New York (1995).

[9] C.J. Budd, S. Chen and R.D. Russell, J. Comp. Phys., 152 (1999) 756-789.

[10] C.J. Budd and G.J. Collins, in Numerical Analysis 1997 [Eds. D. Griffiths, D. Higham and G. Watson], Reserach Notes Math. Sci., vol. 380, Longman, London, pp. 16-36 (1998).

[11] C.J. Budd and G.J. Collins, Appl. Numer. Math., 26 (1998) 23-39.

[12] C.J. Budd, G.J. Collins, W.Z. Huang and R.D. Russell, Phil. Trans. R. Soc. Lond. A, 357 (1999) $1047-1077$.

[13] C.J. Budd, W.Z. Huang and R.D. Russell, SIAM J. Sci. Comp., 357 (1996) 305-327.

[14] C.J. Budd and A. Iserles (Editors), Geomtric Integration: Numerical Solution of Differential Equations on Manifolds, Phil. Trans. R. Soc. Lond. A, 357, pp. 943-1133 (1999).

[15] C.J. Budd, B. Leimkuhler and M.D. Piggott, Scaling invariance and adaptivity, preprint maths9918, Department of Mathematical Sciences, University of Bath, U.K. (1999).

[16] C.J. Budd and M.D. Piggott, The geometric integration of scale invariant ordinary and partial differential equations, preprint maths0005, Department of Mathematical Sciences, University of Bath, U.K. (2000).

[17] G.I. Burde, Q. J. Mech. Appl. Math, 47 (1994) 247-260; 48 (1995) 611-633.

[18] G.I. Burde, J. Phys. A: Math. Gen., 29 (1996) 1665-1983.

[19] P.A. Clarkson, Chaos, Solitons and Fractals, 5 (1995) 2261-2301.

[20] B. Champagne, W. Hereman and P. Winternitz, Comp. Phys. Comm., 66 (1991) 319-340.

[21] P.A. Clarkson and M.D. Kruskal, J. Math. Phys., 30 (1989) 2201-2213.

[22] P.A. Clarkson, D.K. Ludlow and T.J. Priestley, Meth. Appl. Anal., 4 (1997) 173-195.

[23] P.A. Clarkson and E.L. Mansfield, Physica D, 70 (1994) 250-288.

[24] P.A. Clarkson and E.L. Mansfield, SIAM J. Appl. Math., 54 (1994) 1693-1719.

[25] P.A. Clarkson and E.L. Mansfield, Nonlinearity, 7 (1994) 975-1000.

[26] P.A. Clarkson and E.L. Mansfield, Acta Appl. Math., 39 (1995) 245-276.

[27] P.A. Clarkson, E.L. Mansfield, and T.J. Priestley, Math. Comp. Model., 25 (1997) 195-212.

[28] P.A. Clarkson and T.J. Priestley, Stud. Appl. Math., 101 (1998) 389-432.

[29] P.A. Clarkson and P. Winternitz, in The Painlevé Property, One Century Later [Ed. R. Conte], CRM Series in Mathematical Physics, Springer-Verlag, New York, pp. 591-660 (1999).

[30] P.E. Crouch and R. Grossman, J. Nonlinear Sci., 3 (1993) 1-3.

[31] V.A. Dorodnytsyn, in Modern Group Analysis: Advanced Analytical and Computational Methods in Mathematical Physics [Eds. N.H. Ibragimov, M. Torrisi and A. Valenti], Kluwer, Dordrecht, pp. 191-201 (1993).

[32] V.A. Dorodnytsyn, R. Kozlov and P. Winternitz, J. Math. Phys., 41 (2000) 480-504.

[33] M. Fels and P.J. Olver, Acta Appl. Math., 51 (1998) 161-213.

[34] M. Fels and P.J. Olver, Acta Appl. Math., 55 (1999) 127-208.

[35] W.I. Fushchych, W.M. Shtelen, and N.I. Serov, Symmetry Analysis and Exact Solutions of the Equations of Mathematical Physics, Kluwer, Dordrecht (1993).

[36] V.A. Galaktionov, Diff. and Int. Eqns., 3 (1990) 863-874.

[37] A.G. Hansen, Similarity Analyses of Boundary Value Problems in Engineering, Prentice-Hall, Englewood Cliffs (1964).

[38] W. Hereman, in Lie Group Analysis of Differential equations. III. New Trends in Theoretical Developments an Computational Methods [Ed. N.H. Ibragimov], CRC Press, Boca Raton, Chapter XII, pp. 367-413 (1996).

[39] R. Hernandez Heredero, D. Levi and P. Winternitz, J. Phys. A: Math. Gen., 33 (2000) $2685-2695$.

[40] S. Hood, J. Math. Phys., 36 (1995) 1971-1990.

[41] W.Z. Huang, Y. Ren and R.D. Russell, J. Comp. Phys., 112 (1994) 279-290.

[42] E. Hubert, J. Symb. Comput, 29 (2000) 641-662.

[43] P.E. Hydon, Phil. Trans. R. Soc. Lond. A, 456 (2000) 2835-2855.

[44] P.E. Hydon, Symmetry Methods for Differential Equations: A Beginner's Guide, Cambridge Texts in Applied Mathematics, Cambridge University Press, Cambridge (2000).

[45] A. Iserles, in Foundations of Computational Mathematics [Eds. F. Cucker and M. Shub], Springer-Verlag, New York, pp. 180-189 (1997).

[46] A. Iseles and S. Nørsett, Phil. Trans. R. Soc. Lond. A, 357 (1999) 983-1019.

[47] I.S. Krasil'shchik and A.M. Vinogradov, Symmetries and Conservation Laws for Differential Equations of Mathematical Physics, Translations of Mathematical Monographs, vol. 182, American Mathematical Society, Providence, R.I. (1999).

[48] S. Lafortune, L. Martina and P. Winternitz, J. Phys. A: Math. Gen., 33 (2000) 2419-2435; 6431-6446.

[49] D. Levi, J. Negro and M.A. del Olmo, J. Phys. A: Math. Gen., Discrete derivatives and symmetries of difference equations (2000).preprint

[50] D. Levi, S. Trembley and P. Winternitz, J. Phys. A: Math. Gen., 33 (2000) 8507-8523.

[51] D. Levi and P. Winternitz, J. Phys. A: Math. Gen., 22 (1989) 2915-2924.

[52] D. Levi and P. Winternitz, Phys. Lett., 152A (1992) 335-338.

[53] D. Levi and P. Winternitz, J. Math. Phys., 34 (1993) 3713-3740.

[54] W. Magnus, Commun. Pure Appl. Math., VII (1954) 649-673.

[55] E.L. Mansfield, diffgrob2: A symbolic algebra package for analysing systems of PDE using Maple, ftp ftp.ukc.ac.uk, login: anonymous, password: your email address, directory: pub/maths/liz, files: diffgrob2_src.tar.Z, diffgrob2_man.tex.z (1993). 
[56] E.L. Mansfield, Maple Technical Newsletter, 3 (1996) 33-37.

[57] E.L. Mansfield, J. Math. Anal. Appl., 231 (1998) 526-542.

[58] E.L. Mansfield, Algorithms for symmetric differential systems, preprint UKC/IMS/99/34, Institute of Mathematics and Statistics, University of Kent, U.K. Available from

http://www.ukc.ac.uk/ims/maths/people/E.L.Mansfield.html together with supporting software. (1999).

[59] E.L. Mansfield and P.A. Clarkson, J. Symb. Comp., 23 (1997) 517-533.

[60] E.L. Mansfield and P.A. Clarkson, Math. Comp. Simul., 43 (1997) 39-55.

[61] E.L. Mansfield and E. Fackerell, Differential Gröbner Bases, preprint 92/108, Macquarie Univerisity, Sydney, Australia (1992).

[62] E.L. Mansfield, G.J. Reid and P.A. Clarkson, Comp. Phys. Commun., 115 (1998) 460-488.

[63] R.I. McLachlan, SIAM J. Sci. Comp., 16 (1995) 151-168.

[64] R.I. McLachlan, G.R.W. Quispel and N. Robidoux, Phil. Trans. R. Soc. Lond. A, 357 (1999) 1021-1045.

[65] R.I. McLachlan, G.R.W. Quispel and G.S. Turner, SIAM J. Numer. Anal., 35 (1998) 586-599.

[66] R.I. McLachlan and C. Scovel, J. Nonlinear Science, 5 (1995) 233-256.

[67] H. Munthe-Kass, BIT, 35 (1995) 572-287.

[68] P.J. Olver, Appl. Num. Math., 10 (1992) 307-324.

[69] P.J. Olver, Applications of Lie Groups to Differential Equations, Second Edition, Graduate Texts Math. vol. 107, SpringerVerlag, New York (1993).

[70] P.J. Olver, Equivalence, Invariants, and Symmetry, Cambridge University Press, Cambridge (1995).

[71] P.J. Olver and P. Rosenau, Phys. Lett., 114A (1986) 107-112.

[72] P.J. Olver and P. Rosenau, SIAM J. Appl. Math., 47 (1987) 263-275.

[73] P.J. Olver and E.M. Vorob'ev, in Lie Group Analysis of Differential equations. III. New Trends in Theoretical Developments an Computational Methods [Ed. N.H. Ibragimov], CRC Press, Boca Raton, Chapter X, pp. 291-328 (1996).

[74] $\mathrm{M}^{a}$ Concepción Muriel Patino, Reductions and Conservation of Symmetries, PhD Thesis, University of Salamanca, Spain. (1999).

[75] G.R.W. Quispel, H.W. Capel and R. Sahadevan, Phys. Lett., 170A (1992) 379-383.

[76] G.J. Reid, A.D. Wittkopf and A. Boulton, Eur. J. Appl. Math., 7 (1996) 604-635.

[77] J.M. Sanz-Serna and M. Calvo, Numerical Hamiltonian Problems, Chapman and Hall, London (1994).

[78] A. Zanna and H. Munthe-Kaas, in Foundations of Computational Mathematics [Eds. F. Cucker and M. Shub], SpringerVerlag, New York, pp. 434-443 (1997).

Institute of Mathematics and Statistics, University of Kent, Canterbury, CT2 7NF, U.K.

E-mail address: P.A.Clarkson@ukc.ac.uk, E.L.Mansfield@ukc.ac.uk 
[56] E.L. Mansfield, Maple Technical Newsletter, 3 (1996) 33-37.

[57] E.L. Mansfield, J. Math. Anal. Appl., 231 (1998) 526-542.

[58] E.L. Mansfield, Algorithms for symmetric differential systems, preprint UKC/IMS/99/34, Institute of Mathematics and Statistics, University of Kent, U.K. Available from

http://www.ukc.ac.uk/ims/maths/people/E.L.Mansfield.html together with supporting software. (1999).

[59] E.L. Mansfield and P.A. Clarkson, J. Symb. Comp., 23 (1997) 517-533.

[60] E.L. Mansfield and P.A. Clarkson, Math. Comp. Simul., 43 (1997) 39-55.

[61] E.L. Mansfield and E. Fackerell, Differential Gröbner Bases, preprint 92/108, Macquarie Univerisity, Sydney, Australia (1992).

[62] E.L. Mansfield, G.J. Reid and P.A. Clarkson, Comp. Phys. Commun., 115 (1998) 460-488.

[63] R.I. McLachlan, SIAM J. Sci. Comp., 16 (1995) 151-168.

[64] R.I. McLachlan, G.R.W. Quispel and N. Robidoux, Phil. Trans. R. Soc. Lond. A, 357 (1999) 1021-1045.

[65] R.I. McLachlan, G.R.W. Quispel and G.S. Turner, SIAM J. Numer. Anal., 35 (1998) 586-599.

[66] R.I. McLachlan and C. Scovel, J. Nonlinear Science, 5 (1995) 233-256.

[67] H. Munthe-Kass, BIT, 35 (1995) 572-287.

[68] P.J. Olver, Appl. Num. Math., 10 (1992) 307-324.

[69] P.J. Olver, Applications of Lie Groups to Differential Equations, Second Edition, Graduate Texts Math. vol. 107, SpringerVerlag, New York (1993).

[70] P.J. Olver, Equivalence, Invariants, and Symmetry, Cambridge University Press, Cambridge (1995).

[71] P.J. Olver and P. Rosenau, Phys. Lett., 114A (1986) 107-112.

[72] P.J. Olver and P. Rosenau, SIAM J. Appl. Math., 47 (1987) 263-275.

[73] P.J. Olver and E.M. Vorob'ev, in Lie Group Analysis of Differential equations. III. New Trends in Theoretical Developments an Computational Methods [Ed. N.H. Ibragimov], CRC Press, Boca Raton, Chapter X, pp. 291-328 (1996).

[74] $\mathrm{M}^{a}$ Concepción Muriel Patino, Reductions and Conservation of Symmetries, PhD Thesis, University of Salamanca, Spain. (1999).

[75] G.R.W. Quispel, H.W. Capel and R. Sahadevan, Phys. Lett., 170A (1992) 379-383.

[76] G.J. Reid, A.D. Wittkopf and A. Boulton, Eur. J. Appl. Math., 7 (1996) 604-635.

[77] J.M. Sanz-Serna and M. Calvo, Numerical Hamiltonian Problems, Chapman and Hall, London (1994).

[78] A. Zanna and H. Munthe-Kaas, in Foundations of Computational Mathematics [Eds. F. Cucker and M. Shub], SpringerVerlag, New York, pp. 434-443 (1997).

Institute of Mathematics and Statistics, University of Kent, Canterbury, CT2 7NF, U.K.

E-mail address: P.A.Clarkson@ukc.ac.uk, E.L.Mansfield@ukc.ac.uk 